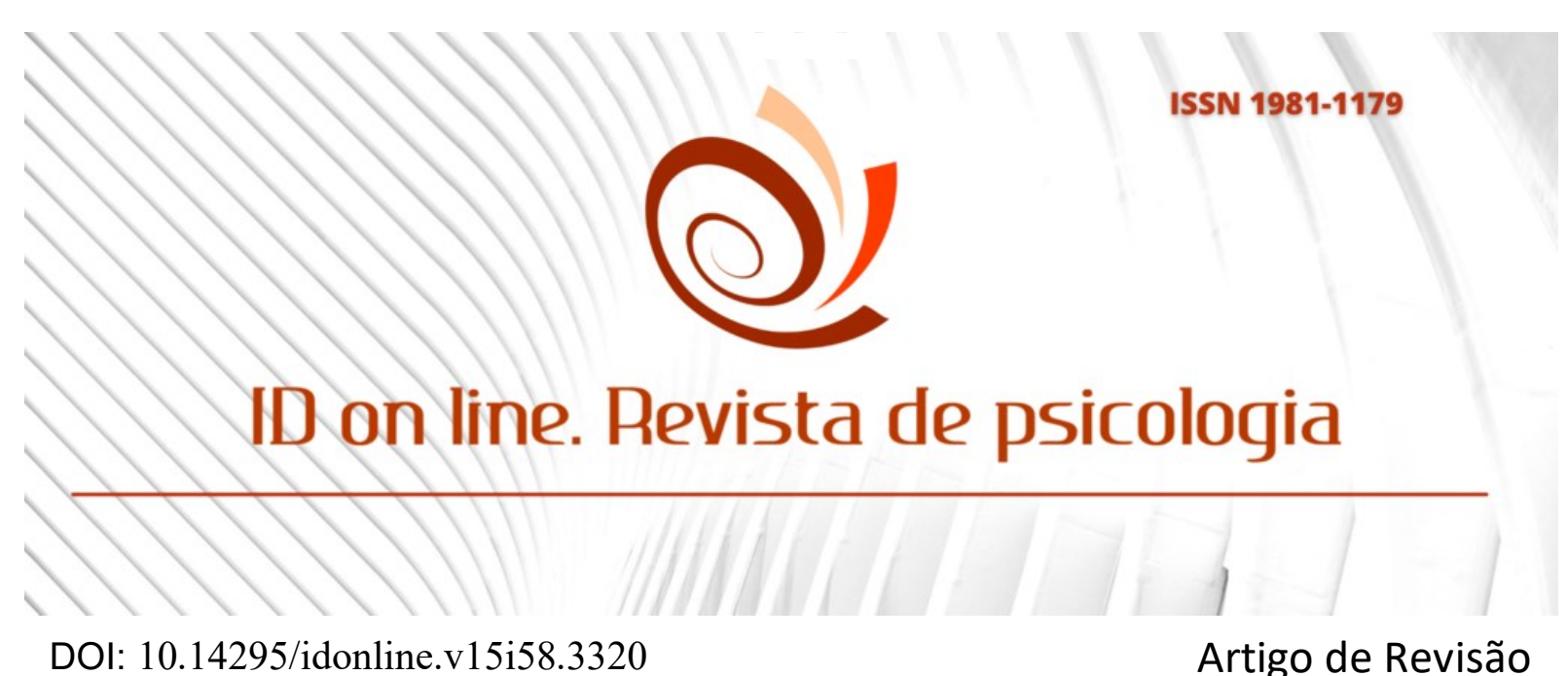

DOI: $10.14295 /$ idonline.v15i58.3320

Artigo de Revisão

\author{
O Impacto Psicossocial da Acne Vulgar \\ Luísna Gabriela Aguiar Lobo de Resende ${ }^{\text {; }}$ Gabriel Cardoso Oliveira da Silva²; \\ Érica Carvalho Caldas ${ }^{3}$
}

\begin{abstract}
Resumo: A acne é uma afecção cutânea comum, que atinge cerca de $80 \%$ da população em alguma fase da vida. Além da parte estética, a acne abrange consequências psicológicas e sociais que podem afetar a qualidade de vida, saúde psíquica e a autoestima dos pacientes. O presente estudo de revisão narrativa bibliográfica, investigou os aspectos principais do impacto psicossocial em pessoas com acne vulgar. Constatou-se que vários danos são gerados para os acometidos por essa patologia, entre eles estão: ansiedade, fobia social, depressão, insegurança, timidez, sintomas obsessivos compulsivos e transtorno dismórfico corporal. Tendo em vista os resultados apontados, confirmou-se que o tratamento adequado e efetivo apresenta grande melhora no quadro dos pacientes. Diante dos malefícios que a acne promove para vida da população é necessário compreendê-la como uma doença e não apenas como uma disfunção estética.
\end{abstract}

Palavras-Chave: acne vulgar, fatores psicológicos, psicossocial e acne.

\title{
The Psychosocial Impact of Acne Vulgaris
}

\begin{abstract}
Acne is a common skin condition that affects about $80 \%$ of the population at some stage of life. In addition to the aesthetic part, acne includes psychological and social consequences that can affect the quality of life, mental health, and self-esteem of patients. The present study of a bibliographic narrative review investigated the main aspects of the psychosocial impact on people with acne vulgaris. It was found that several damages are generated for those affected by this pathology, including anxiety, social phobia, depression, insecurity, shyness, obsessive-compulsive symptoms and body dysmorphic disorder. In view of the above results, it was confirmed that the adequate and effective treatment presents a great improvement in the patients' condition. In view of the harm that acne causes to the population's life, it is necessary to understand it as a disease and not just an aesthetic dysfunction.
\end{abstract}

Keywords: acne vulgaris, psychological factors, psychosocial and acne.

\footnotetext{
${ }^{1}$ Acadêmica de Estética e Cosmética. Instituição: Instituição de Educação Superior de Brasília - IESB. Brasília DF, ORCID: 0000-0001-7008-6194. luisna.resende@gmail.com

${ }^{2}$ Químico, Doutor em química pela universidade de Brasília-UNB- Brasília-DF, ORCID: 0000-0002-9005-5675. gcardosilva@gmail.com

${ }^{3}$ Fisioterapeuta, Mestre em Gerontologia pela Universidade Católica de Brasília - UCB. Instituição: Instituição de Educação Superior de Brasília -. Brasília-DF, ORCID: 0000-0002-6928-3155. caldaserica@gmail.com
} 


\section{Introdução}

A acne vulgar é uma afecção cutânea que se desenvolve nos folículos pilossebáceos. Cerca de $80 \%$ da população é afetada em alguma fase da vida, principalmente na adolescência. As regiões mais acometidas são a face e o tronco (TASOULA et al., 2012). Seu agravamento pode estar relacionado a fatores hormonais, genéticos, emocionais, estresse e alimentação. Sendo dessa forma, considerada uma doença multifatorial crônica (DE ARAÚJO; DELGADO; MARÇAL, 2011).

Quanto mais alto o grau da acne, maior a insegurança e transtornos gerados para vida social do paciente. Além, da grande influência das mídias sociais na atualidade, que estabelece a busca incessante pela perfeição, acarretando consequências diretas no vestuário, relacionamentos, lazer e vida sexual (NEVES et al., 2021).

As sequelas e lesões provocadas pela acne impactam de forma psicossocial a vida da maioria das pessoas, levando-as muitas vezes a desenvolverem ansiedade, insegurança, fobia social, timidez, sintomas obsessivos compulsivos e transtorno dismórfico corporal (OZTURK et al., 2013).

Outra alteração que pode ser desenvolvida pela acne é a depressão, tendo em vista que, essa patologia causa maiores efeitos adversos no humor e na saúde mental em relação à outras doenças cutâneas (LANGER e PRIM, 2020).

Em um estudo realizado em 2010, foi constatado que 8,8\% dos pacientes apresentaram depressão. No mesmo estudo, foi relatado que mulheres $(10,6 \%)$ com a respectiva patologia acabam sofrendo duas vezes mais depressão do que os homens $(5,3 \%)$ (TASSINARY; SINIGAGLIA; SINIGAGLIA, 2019, p.66).

O presente trabalho tem como objetivo evidenciar por meio de artigos, os impactos psicossociais em pacientes com acne vulgar, visto que, a acne afeta grande parte da população, causando diversos desconfortos. Torna-se necessário reconhecer as consequências dessas lesões e dos cuidados com a saúde cutânea e psíquica.

\section{Metodologia}

A pesquisa é composta por uma revisão narrativa de literatura realizada com base em artigos científicos do banco de dados do Google Acadêmico, Pubmed e Scielo, nos idiomas 
português e inglês. Foram usadas como palavras chaves os descritivos: "acne vulgar", "fatores psicológicos", "psicossocial” e "acne". As buscas sobre o tema ocorreram de agosto a novembro de 2021. Os artigos utilizados foram filtrados em materiais publicados nos últimos 17 anos.

\section{Acne}

Propionibacterium acnes, vulgarmente conhecida com espinha é uma bactéria anaeróbica, grampositiva. Prolifera-se no ducto folicular, gerando uma irritação na parede do ducto e impulsionando a queratinização em razão da hidrólise dos triglicerídeos do sebo (MONTAGNER e COSTA, 2010).

Há múltiplas escalas de classificação de acne embasadas em diversos aspectos da doença. Uma das escalas mais utilizadas, é a de severidade da acne, que avalia as lesões faciais classificando-as em graus, e variando de 1 a 5; sendo: (LÓPEZ-ESTEBARANZ et al., 2017)

- Grau I considerada não inflamatória ou comedoniana, com a presença de comedões abertos e fechados.

- Grau II, observa-se uma maior presença de comedões, pápulas e pústulas. Esse grau é chamado de pápulo-pustulosa.

- Na acne de grau III, cientificamente chamada de nódulo-cística, existem uma maior presença de nódulos e cistos, comumente atinge várias regiões da fase.

- No grau IV, tem a presença da acne conglobata, caracterizada pela formação de múltiplos nódulos inflamatórios, abscessos e fístulas.

- O grau $\mathrm{V}$, é considerado o mais raro e consequentemente o mais grave, pois além da presença de múltiplos nódulos inflamatórios, abscessos e fístulas, pode ser acompanhado de febre, leucocitose e artralgia (DE OLIVEIRA; TORQUETTI; NASCIMENTO, 2020).

Em relação a etnia, apresenta predominância na raça caucasiana, sendo menos intensa em pessoas de etnia asiática e negra. Em relação ao gênero, o sexo masculino manifesta de forma mais grave a patologia. A prevalência nos homens, está ligada à influência androgênica. A assiduidade populacional aumenta com a idade e com o histórico familiar (COSTA; ALCHORNE; GOLDSCHMIDT, 2008).

Nas mulheres a causa mais comum está associada a uma resposta alterada dos receptores androgênicos, tendo como consequência mudanças fisiológicas e no ciclo 
menstrual, associadas a lesões inflamatórias e aumento da produção de sebo. As lesões de acne principalmente no período pré-menstrual e menstrual, podem ser aumentadas em até 70\% (ADDOR e SCHALKA, 2010).

A alimentação e o estilo de vida influenciam diretamente na patogênese da acne. A ingestão de alimentos com alto índice glicêmico como: ultra processados, leite desnatado e alimentos ricos em amido, juntamente com estresse fisiológico e tabagismo afetam a severidade da acne (OGE'; BROUSSARD; MARSHALL, 2019).

\section{Acne e o Impacto Psicossocial}

Determinados estudos constataram que os indivíduos com acne desenvolvem com frequência alterações na saúde psíquica; sendo que essa probabilidade tende a aumentar diante da severidade da patologia repercutindo na vida social. Segundo o mesmo autor, o impacto psicossocial pode ser comparado com o investigado em pessoas com epilepsia, asma, diabetes e artrite (DIAS, 2014),

No período da adolescência, estão presentes muitos distúrbios psicológicos, ocasionando a vulnerabilidade desses indivíduos. As modificações corporais representam uma fase propícia ao stress psicossocial e à acne. Entretanto, na fase adulta, pressupõe-se que existam 40\% de comorbilidades psíquicas (TEIXEIRA; VIEIRA; FIGUEIREDO, 2012). Em 2009 um estudo mostrou que a depressão é 2 a 3 vezes mais predominante nas pessoas com a doença, quando comparada a população geral (UHLENHAKE et al., 2010).

Observa-se um maior índice de depressão nos adultos com acne do que na população jovem, sobretudo, entre o público feminino. As mulheres adultas entre 25 a 40 anos, apresentam vulnerabilidade maior devido ao stress adicional, maternidade, exigências profissionais, alterações hormonais e envelhecimento (UHLENHAKE et al., 2010).

A depressão e a ansiedade podem ser consideradas fatores secundários, ou seja, podem estar associados a outros fatores tais como: isolamento social, distorção da imagem, autoestima, suporte familiar, patologia psiquiátrica subjacente e mecanismo de coping. Considera-se que o impacto psicológico da acne, é individualizado e incalculável (TEIXEIRA; VIEIRA; FIGUEIREDO,2012).

Para Neves (2016), a depressão é caracterizada pela perda de motivação e autoestima, e associa-se à baixa aptidão para atingir os objetivos relevantes para o indivíduo. Engloba disforia, desânimo, desvalorização da vida, autodepreciação, falta de interesse e inércia. Pode 
ser identificada como uma alteração obstinada do humor, baixa autoestima, sentimentos de desvalorização pessoal e perda da disposição (Yang, et al., 2014).

Neves (2016) relata ainda que o impacto da depressão e o suicídio em pessoas com acne varia de acordo com a idade, etnia, gênero e cultura. Apesar da ligação entre a acne e o suicídio não ser significativa perante as estatísticas, observa-se uma tendência de aumento do risco de suicídio entre os pacientes com a acne, principalmente entre os pacientes que possuem a doença de forma moderada a grave.

O suicídio, caracterizam-se pelo sinal de estresse emocional. Ocorre geralmente em pacientes com elevado nível de depressão (PICARDI; LEGA; TAROLLA, 2013). É uma das causas de morte mais frequentes mundialmente, com cerca de 1 milhão de óbitos anuais. As taxas de mortalidade mundial são de 16 óbitos por cada 100.000 habitantes. Sendo o suicídio considerado um problema de saúde pública muito delicado e grave (MACHADO e SANTOS, 2015).

Os índices de ideação suicidária são mais proeminentes nos pacientes com acne do que nos pacientes em geral, e a elevada depressão nesses pacientes está associada à maior frequência de ideação do suicídio, principalmente nas mulheres, devido à cobrança que a sociedade impõe para que alcancem um padrão análogo à perfeição (NEVES, 2016).

A ideia de suicídio não deve ser vista apenas como a tentativa, mas pode ser representada por diversos fatores como: saturação da vida, desejo da morte, ideação do suicídio, planos de suicídio, tentativa de suicídio. Por isso, considerara acne como um fator apenas estético se torna perigoso (PICARDI; MAZZOTTI; PASQUINI, 2006).

Kurtalic et al. (2010) mencionou outra alteração; a ansiedade, que quando procedente da acne é superior à registada em pacientes com câncer de pele. Essa determinada situação provém da importância que a imagem possui, sobretudo para os adolescentes. Entretanto, as questões na qual a pele se encontra, pode acarretar consequências negativas na qualidade de vida dos jovens (NEVES, 2016).

Uma pesquisa constatou que $70 \%$ das pessoas entrevistadas sofriam rejeição social por ter acne ativa. Experiência como essas podem ser consideradas impactantes para o desenvolvimento de danos emocionais futuros. Quando questionados em relação às questões de sensibilidade social, com base na opinião que as pessoas constroem acerca dos indivíduos com acne; os resultados demonstraram que os entrevistados apresentaram grandes preocupações referentes a forma de como são vistos pela sociedade (KREJCIMANWARING et al., 2006). 
A fobia social é um transtorno psiquiátrico que ocasiona sofrimento e perda de oportunidade considerável para a pessoa atingida. Indivíduos com fobia social apresentam um medo demasiado de serem vistos, portando-se de um modo humilhante pela demonstração de ansiedade ou de execução inapropriada e consequentemente da condenação ou rejeição por parte das outras pessoas (ANGÉLICO e LOUREIRO, 2012).

Indivíduos com predisposição a sentir raiva, por exemplo, apresentam uma qualidade de vida menor e consequentemente menor satisfação com o tratamento em relação aos pacientes com menos sentimentos de raiva (RAPP el., 2004).

Fatores estressores também causam as disfuncionalidades do organismo, como a acne. O estresse atua como uma resposta necessária da natureza humana para a sobrevivência. Todavia, são inúmeros os efeitos nocivos do estresse na saúde, principalmente causados pelos fatores estressores crônicos e de longo prazo (DHABHAR, 2013).

Além das alterações causadas pela doença e de fatores psicológicos já citadas anteriormente, certos comportamentos e atitudes são observados como características emocionais de risco, como apresentar grande desconforto em manter contacto visual, irritabilidade, discurso limitado, comportamentos compulsivos, relações sociais instáveis e descuido com a higiene pessoal (FRIED et al., 2006).

\section{Tratamento}

O tratamento efetivo da acne tem uma importância significativa, pois é responsável por amenizar os impactos psicossociais, aumento assim a autoconfiança, autoestima e melhoria da imagem social (TAN, 2004).

Os tratamentos são realizados de acordo com o grau de acometimento da patologia. As terapêuticas estéticas apresentam como finalidade reduzir as inflamações e a seborreia, ação comedolítica e melhorando as hiperpigmentações pós inflamatórias e as cicatrizes. É necessário uma anamnese rigorosa e exames referentes ao estado do paciente, antecedentes sistêmicos, comorbidades, emocional e alergias presentes (PEREIRA; COSTA; SOBRINHO, 2019). A seguir, discorreremos sobre alguns dos recursos estéticos realizados para o tratamento das acnes. 


\section{Limpeza de pele}

Tem como objetivo a higienização profunda da pele, removendo assim todas as impurezas do local. (OLIVEIRA; TOQUETTI; NASCIMENTO, 2020). Alguns dos princípios ativos utilizados na limpeza de pele são: ácido salicílico, taninos, hamamelis, óleo de melaleuca, eucalipto e $\alpha$-hidroxiácidos (PEREIRA; COSTA; SOBRINHO, 2019).

\section{Peelings químicos}

Os peelings químicos são constituídos por uma esfoliação responsável pela promoção da injúria à pele, provocando danos controlados. Ocorre, logo em seguida, a liberação de citocinas e mediadores da inflamação, tendo como resultado o espessamento da epiderme, depósito de colágeno, aumento do volume dérmico e reorganização dos elementos estruturais. A classificação mais utilizada em reação aos peelings são: muito superficial, atingindo a camadas córnea e granulosa; superficial, atingindo toda a epiderme; médio, chegando até a derme papilar e profundo, atingindo a derme reticular (BAGATIN; HASSUN; TALARICO, 2009).

O peeling químico é utilizado no tratamento de acne vulgar e suas sequelas. Entre os ácidos mais utilizados estão presentes: o ácido salicílico, ácido retinóico, ácido glicólico, ácido pirúvico, ácido mandélico, ácido láctico, ácido tricloroacético, solução de Jessner e o fenol. O peeling ideal para cada tipo de pele é selecionado de acordo com o fototipo cutâneo, dado pela classificação de Fitzpatrick (PERIC et al., 2011).

\section{A Luz Intensa Pulsada (LIP)}

Existem dois mecanismos de ação da luz intensa pulsada que promovem a melhora da acne ativa. O primeiro efeito é o fotodinâmico pela luz visível e pelo espectro UV que quando absorvido pelas porfirinas produzidas pela Propionibacterium acnes (P. acnes), que resulta em formação de radicais livres de oxigênio encarregado pelo efeito bactericida. Já o segundo mecanismo é baseado na fototermólise seletiva dos vasos sanguíneos que são responsáveis por nutrir a glândula sebácea, reduzindo o fluxo sanguíneo,e a taxa de secreção da glândula sebácea diminui (KALIL; REINEHR; DE MATTOS, 2017). 


\section{LED}

Os light emitting diodes (LEDs) são diodos semicondutores submetidos a uma corrente elétrica, capazes de emitirem luzes que podem ser utilizadas na fototerapia (ESTRELA et al., 2014). As frequências específicas dos LEDs, como a de $415 \mathrm{~nm}$ (azul), 633 $\mathrm{nm}$ (vermelho) e $830 \mathrm{~nm}$ (infravermelho), apresentam significativos resultados para o tratamento de acne vulgar (PEREIRA; COSTA; SOBRINHO, 2019).

A finalidade da fototerapia para acne vulgar necessita das características da bactéria $P$. acnes, em que as porfirinas produzidas pelas bactérias absorvem luz no espectro visível. A que possui mais abundância é a coproporfirina III, que tem ápice de absorção em $415 \mathrm{~nm}$, e as espécies reativas de oxigênio produzidas por fotoexcitação são capazes de eliminar a $P$. acnes (KIM et al., 2011).

\section{Laser de diodo de $1.450 \mathrm{~nm}$}

A utilização do laser de diodo de $1.450 \mathrm{~nm}$ tem sido considerado uma terapia auxiliar para o tratamento de acne vulgar e suas sequelas, com remissão a longo prazo. Apresentando eficácia superior aos tratamentos tópicos ou com o uso de antibióticos (ALAM et al., 2011).

\section{Microdermoabrasão}

Microdermoabrasão é uma técnica muito utilizada em tratamentos de diferentes afecções cutâneas, incluindo acne e suas sequelas. Para a técnica leva-se em consideração: A pressão utilizada, quantidade de microcristais jateados e número de sessões. Essa terapia tem como resultado a melhoria das irregularidades da pele, nivelamento tecidual e estimulação proteica adequada na região depressiva (PEREIRA; COSTA; SOBRINHO, 2019).

\section{Microagulhamento}

O microagulhamento, é responsável por estimular o colágeno através dos microtraumas gerados na derme superficial. Podem ser adicionados fatores de crescimento, que estimulam a neocolagênese. Provocando a formação de um novo tecido conjuntivo que preenche as regiões de cicatrizes atróficas e induzem a repigmentação local (PEREIRA; COSTA; SOBRINHO, 2019). 


\section{Aromoterapia}

Aromaterapia é uma terapêutica que tem como objetivo o bem-estar físico e mental, promove o relaxamento, a diminuição do estresse e auxiliando tratamento de diversas patologias estéticas. No Brasil tem crescido consideravelmente a procura por essa Prática Integrativa e Complementar (PIC). Ela pode ser associada a outras terapêuticas, como por exemplo, a argiloterapia na área da estética (CORRÊA; SCHOTTEN; MACHADO, 2010) e (CRUZ e DA PAIXÃO, 2021).

Um dos óleos essenciais mais utilizado para o tratamento dessa patologia é o de melaleuca, que possui propriedades bactericidas, cicatrizantes, anti-inflamatória, anti-séptica, antifúngica, antiviral, imunoestimulante, febrífuga, inseticida, diaforética, anticaspa, parasiticida, germicida e desinfetante (CRUZ e DA PAIXÃO, 2021).

\section{Argiloterapia}

As argilas são de origem terrosa e sua formação química é feita por silicatos, ferro, magnésio e hidratados de alumínio. Cada argila possui um fim terapêutico específico e as cores contribuem para esta diferenciação (TOYOKI e DE OLIVEIRA, 2015).

As indicações ideais são as argilas verde e branca em virtude de que seus ativos auxiliam na cicatrização, regeneração tecidual, agem no controle da secreção sebácea, ação adstringente, calmante e são antissépticos naturais. Uma forma de potencializar a máscara de argila é adicionando algumas gotas de Óleo de Melaleuca (SOUZA, 2009).

\section{Nutricosméticos}

Os nutricosméticos são como suplementos nutricionais que ajudam a cuidar do aspecto da pele, unhas e cabelos (DINI et al., 2019). Os nutricosméticos são derivados de alimentos, como as vitaminas, minerais, óleos, proteínas e extratos (BATTISTI, 2013).

Estudos analisaram que o ômega 3 e os probióticos atuam como principais nutricosméticos coadjuvantes para o tratamento de acne, melhorando consideravelmente as inflamações (SALVADOR e CECHINEL-ZANCHETT, 2019). 


\section{Resultados e Discussão}

Essa pesquisa é de natureza básica, tendo como objetivo primordial corroborar com estudos que demostraram os impactos psicossociais em pacientes que apresentaram a acne vulgar. O estudo é de cunho bibliográfico, do tipo narrativa com a necessidade de se explorar informações, sem a necessidade de se esgotar as fontes de estudo, por se tratar de uma afecção que altera a pele visualmente e psicologicamente, sendo essa de cunho subjetivo. Foram realizadas diversas pesquisas em materiais já publicados como artigos científicos, e foi encontrado um total de 7 artigos entre os anos de 2008 e 2021.

A análise dos artigos foi descrita em duas tabelas evidenciando os resultados deparados em estudos publicados que demonstraram o impacto da acne de forma psicossocial, aclarando as conclusões de cada autor.

A tabela 1, explana os artigos mencionados e os resultados evidenciados.

\section{Tabela 1- artigos, autores, referencias e conclusões}

\begin{tabular}{|c|c|c|c|}
\hline Título do artigo & Autores e ano & Periódico & Conclusões \\
\hline $\begin{array}{l}\text { O impacto da acne vulgar } \\
\text { na qualidade de vida e } \\
\text { saúde psíquica em jovens } \\
\text { adolescentes na Grécia. } \\
\text { Resultados de uma } \\
\text { pesquisa populacional }\end{array}$ & (TASOULA,et al., 2012) & $\begin{array}{l}\text { Anais Brasileiros } \\
\text { de Dermatologia }\end{array}$ & $\begin{array}{l}\text { Conclui-se que com os } \\
\text { sintomas da acne a saúde } \\
\text { psicológica e principalmente a } \\
\text { autoestima dos atingidos são } \\
\text { impactadas. Deve ser levada } \\
\text { em consideração a avaliação } \\
\text { da qualidade de vida dos } \\
\text { adolescentes com acne, pois } \\
\text { os mesmos podem ter } \\
\text { comprometimentos futuros. }\end{array}$ \\
\hline $\begin{array}{lll}\text { Impacto psicossocial da } \\
\text { acne: influência } & \text { do } \\
\text { tratamento } & \text { com } \\
\text { isotretinoína } & \end{array}$ & (DIAS,2014) & $\begin{array}{c}\text { Instituto de } \\
\text { ciências } \\
\text { biomédicas Abel } \\
\text { Salazar } \\
\text { universidade do } \\
\text { Porto }\end{array}$ & $\begin{array}{l}\text { Evidenciou que a acne tem um } \\
\text { impacto psicossocial e } \\
\text { emocional grande na vida dos } \\
\text { acometidos pela patologia. } \\
\text { Constatou também que o } \\
\text { tratamento ajuda a melhorar o } \\
\text { bem-estar dos doentes. }\end{array}$ \\
\hline $\begin{array}{l}\text { Acne vulgar e bem-estar } \\
\text { em acadêmicos de } \\
\text { medicina }\end{array}$ & $\begin{array}{l}\text { (RIBAS; } \\
\text { OLIVEIRA;RIBEIR, 2008) }\end{array}$ & $\begin{array}{l}\text { Anais Brasileiros } \\
\text { de Dermatologia }\end{array}$ & $\begin{array}{l}\text { A maioria dos pacientes } \\
\text { entrevistados manipulavam } \\
\text { com frequência as lesões, } \\
\text { sentiam desgosto por ter acne, } \\
\text { apresentavam ansiedade, } \\
\text { insatisfação quanto à } \\
\text { aparência física e medo de a } \\
\text { acne nunca cessar. }\end{array}$ \\
\hline
\end{tabular}




\begin{tabular}{|c|c|c|c|}
\hline $\begin{array}{l}\text { A vivência da Acne e } \\
\text { assuas consequências } \\
\text { psicológicas }\end{array}$ & (NEVES et al.,2021) & $\begin{array}{l}\text { Brazilian Journal } \\
\text { of Health Review }\end{array}$ & $\begin{array}{l}\text { Esse estudo mostrou que a } \\
\text { vivência de pessoas com acne } \\
\text { leva a uma perda da qualidade } \\
\text { de vida e que a acne está } \\
\text { associada a níveis de } \\
\text { depressão, estresse } \\
\text { ansiedade. }\end{array}$ \\
\hline $\begin{array}{l}\text { Impacto na saúde mental } \\
\text { do paciente com acne } \\
\text { grave/moderada em uso } \\
\text { da isotretinoína oral: } \\
\text { Estudo qualitativo }\end{array}$ & $\begin{array}{l}\text { (SARAIVA e DO } \\
\text { EGYPTO, 2021) }\end{array}$ & $\begin{array}{l}\text { Research, Society } \\
\text { and Development }\end{array}$ & $\begin{array}{l}\text { Diante desse } \\
\text { observou-se sentimentos } \\
\text { muito negativos em pessoas } \\
\text { com acne grave; como: } \\
\text { insegurança, baixa autoestima, } \\
\text { introspecção e restrições } \\
\text { cotidianas. A acne além de } \\
\text { prejudicar a saúde da pele } \\
\text { atinge o psicológico e gera } \\
\text { problemas com a auto } \\
\text { imagem. }\end{array}$ \\
\hline
\end{tabular}

Fonte: dados da Pesquisa.

Tabela 2 - Artigos, autores, metodologia e resultados

\begin{tabular}{|c|c|c|c|}
\hline $\begin{array}{l}\text { Título do artigo, } \\
\text { autor e ano }\end{array}$ & Metodologia & Amostra & Principais descobertas \\
\hline $\begin{array}{l}\text { A vivência da Acne e } \\
\text { as suas consequências } \\
\text { psicológicas } \\
\text { (NEVES et al., 2021) }\end{array}$ & $\begin{array}{l}\text { Aplicação de um } \\
\text { questionário de } \\
\text { autopreenchimento }\end{array}$ & 1.666 & $\begin{array}{l}\text { - } 73 \% \text { consideram que a acne é algo } \\
\text { negativo ou muito negativo para si. } \\
\text { - } 80 \% \text { procuram informação sobre a } \\
\text { acne } \\
\text { - } 71 \% \text { dos respondentes seguem à risca } \\
\text { os cuidados com a pele que lhe } \\
\text { indicaram para a Acne. } \\
\text { - } 80 \% \text { dos respondentes que possuem } \\
\text { acne e estão em tratamento, } \\
\text { consideram o mesmo como eficaz. } \\
\text { - } 84 \% \text { dos respondentes que já tiveram } \\
\text { acne considera que o tratamento foi } \\
\text { eficaz. } \\
\text { - } 44 \% \text { dos respondentes usam } \\
\text { maquilhagem de modo a esconder a } \\
\text { Acne. }\end{array}$ \\
\hline Acne vulgar e bem- & Questionário & 100 & $\begin{array}{l}-77 \% \text { manipulavam com frequência as } \\
\text { lesões }\end{array}$ \\
\hline
\end{tabular}

361 Id on Line Rev. Psic. V.15, N. 58, p. 351-367, Dezembro/2021 - Multidisciplinar. ISSN 1981-1179

Edição eletrônica em http://idonline.emnuvens.com.br/id 


\begin{tabular}{|c|c|c|c|}
\hline $\begin{array}{l}\text { estar em acadêmicos } \\
\text { de medicina } \\
\text { (RIBAS;OLIVEIRA; } \\
\text { RIBEIR, 2008) }\end{array}$ & padronizado & $\begin{array}{c}\text { (Entre esses } \\
100 \\
\text { acadêmicos, } 50 \\
\text { possuíam acne) }\end{array}$ & $\begin{array}{l}\cdot 73 \% \text { sentiam desgosto por ter acne } \\
\text {-A ansiedade estava presente em } 50 \% \\
\text { dos casos } \\
\cdot 41,6 \% \text { apresentavam insatisfação } \\
\text { quanto à aparência física } \\
\cdot 35,4 \% \text { tinham medo da acne nunca } \\
\text { cessar } \\
\cdot 35,4 \% \text { sentiam aversão a se olhar no } \\
\text { espelho } \\
\cdot 10,4 \% \text { apresentavam constrangimento } \\
\text { social pela aparência física }\end{array}$ \\
\hline $\begin{array}{l}\text { O impacto da acne } \\
\text { vulgar na qualidade de } \\
\text { vida e saúde psíquica } \\
\text { em jovens } \\
\text { adolescentes na } \\
\text { Grécia. Resultados de } \\
\text { uma pesquisa } \\
\text { populacional } \\
\text { (TASOULAet al., } \\
2012\end{array}$ & $\begin{array}{l}\text { Questionário com } 33 \\
\text { itens para as } \\
\text { mulheres e } 31 \text { itens } \\
\text { para os homens }\end{array}$ & 1531 & $\begin{array}{l}\text { - O estresse auto-relatado pelos alunos } \\
\text { com acne estava presente em } 55,4 \% \\
\cdot 46,8 \% \text { possuíam baixa auto-estima } \\
\cdot 31,4 \% \text { apresentavam sentimentos de } \\
\text { desmerecimento provocado pela acne } \\
\cdot 26,8 \% \text { dos adolescentes com a } \\
\text { respectiva patologia sentiram que eles } \\
\text { eram afetados pelas atitudes da família, } \\
\text { amigos e professores por causa de sua } \\
\text { doença de pele } \\
\cdot 21,4 \% \text { sentiam-se afetados na sua vida } \\
\text { escolar e atividades pessoais, enquanto } \\
19.4 \% \text { eram afetados nos seus } \\
\text { passatempos devido à acne } \\
\cdot 19,2 \% \text { dos estudantes eram afetados } \\
\text { nas suas vidas pessoal e social, } \\
\text { especialmente na construção de seus } \\
\text { relacionamentos }\end{array}$ \\
\hline $\begin{array}{lr}\text { Qualidade de } & \text { vida, } \\
\text { autoestima e } & \text { fatores } \\
\text { psicossociais } & \text { em } \\
\text { adolescentes } & \text { com } \\
\text { acne vulgar } & \\
\text { (VILAR; FILHO; } \\
\text { SANTOS, 2015) }\end{array}$ & $\begin{array}{c}\text { Aplicação do } \\
\text { questionário e foram } \\
\text { submetidos à } \\
\text { avaliação clínica da } \\
\text { pele }\end{array}$ & 355 & $\begin{array}{l}\cdot 58 \% \text { tinham medo de que a acne } \\
\text { nunca cesse } \\
\cdot 52,1 \% \text { apresentavam angústia por ter } \\
\text { acne } \\
\cdot 48,3 \% \text { consideravam-se pessoas } \\
\text { estressadas } \\
\cdot 23,3 \% \text { dos entrevistados com acne não } \\
\text { gostavam da própria aparência ao se } \\
\text { olhar no espelho } \\
\cdot 21,8 \% \text { tinham medo de encontrar } \\
\text { pessoas pela primeira } \\
\cdot 19,2 \% \text { apresentavam medo em ser } \\
\text { fotografados }\end{array}$ \\
\hline
\end{tabular}

Fonte: dados da Pesquisa.

Diante dos artigos citados, compreende-se que a acne traz impactos consideráveis para a vida dos pacientes. Muitas vezes, estes apresentam sentimentos negativos como; 
insegurança, desgosto, estresse e vergonha. Sensações como estas refletem no despenho acadêmico, vida pessoal, bem-estar emocional e relacionamentos interpessoais.

Notou-se a procura por maquiagem com o objetivo de camuflar as lesões, trazendo assim um bem-estar para maioria das pessoas. O mercado vem revolucionando com a maquiagem mineral, pois a mesma não contém talco, ingredientes comedogênicos e alergênicos, sendo bem positiva para os pacientes com acne (OLIVEIRA e FERREIRA, 2017).

Deve-se enfatizar, o temor em relação ao não desaparecimento da acne, pois ele é muito citado na literatura. O medo da patologia nunca cessar foi um dos mais mencionados, podendo levar a manipulação das lesões e tratamentos inapropriados, o que acaba persistindo a doença (TEIXEIRA e FRANÇA, 2007).

A manipulação de forma inadequada das pessoas com acne foi mencionada nos artigos. Entretanto, ao se manusear a acne, vários ricos à saúde podem ser gerados como infecções, devido a contaminações, principalmente com advento da sujidade das unhas; podendo causar hiperpigmentação pós-inflamatória e cicatrizes. Tassinary; Sinigaglia; Sinigaglia (2019 p.75) mencionam que a presença de cicatrizes de acne pode gerar fatores emocionais e psicologicamente angustiantes para os pacientes. Além disso, é um fator de risco para o suicídio, e, pode estar ligado a ansiedade, depressão, menor desempenho acadêmico e ao desemprego, dentre outros.

Um outro problema ocasionado pela manipulação frequente das acnes é a celulite facial. Que pode ter como início um processo infeccioso gerado pelo manuseio das lesões, tornando assim a porta de entrada para bactérias, responsáveis pela difusão do processo inflamatório para o plano subcutâneo (TONIOLLO; TERADA; JUNIOR, 2016).

\section{Conclusão}

Diante dos estudos analisados, constatou-se que a vida dos pacientes com acne é significativamente atingida, levando-os à perda da vivência social qualitativa e podendo estar associada a quadros de depressão, estresse, ansiedade e fobia social. Quanto maior o grau de acometimento da patologia, mais elevados serão os impactos psicossociais.

É imprescindível que se tenha uma assistência médica e psicológica diante de uma doença que afeta certa de $80 \%$ da população, visto que os tratamentos para a acne, demandam 
acompanhamento de especialistas e recursos cosméticos, que na maioria das vezes são de custos elevados, dificultando a sua utilização.

É necessário se pensar na adoção de medidas de inclusão e definição de mecanismos que possam inseri-la no contexto de uma doença a ser assistida adequadamente pelos órgãos de saúde e equipe multidisciplinar, deixando de ser considerada apenas uma questão de estética.

\section{Referências}

ADDOR, Flavia Alvim Sant'Anna; SCHALKA, Sergio. Acne da mulher adulta: aspectos epidemiológicos, diagnósticos e terapêuticos. Anais Brasileiros de Dermatologia, v. 85, p. 789-795, 2010.

ALAM M, GLADSTONE HB, TUNG R. Dermatologia Cosmética: Requisitos em Dermatologia. Rio de Janeiro: Elsevier, 2011.

ANGÉLICO, Antonio Paulo; CRIPPA, José Alexandre S.; LOUREIRO, Sonia Regina. Utilização do Inventário de Habilidades sociais no diagnóstico do Transtorno de Ansiedade Social. Psicologia: Reflexão e Crítica, v. 25, p. 467-476, 2012.

ASCHBACHER, Kirstin et al. Bom estresse, mau estresse e estresse oxidativo: percepções da reatividade antecipatória do cortisol. Psychoneuroendocrinology , v. 38, n. 9, pág. 1698$1708,2013$.

BAGATIN, Ediléia; HASSUN, Karime; TALARICO, Sérgio. Revisão sistemática sobre peelings químicos. Surgical\&CosmeticDermatology, v. 1, n. 1, p. 37-46, 2009.

BATTISTI, Betina Zimmermann et al. Nutricosméticos no processo de envelhecimento de mulheres. Dissertação (Pós-Graduação em Envelhecimento Humano)- Universidade de Passa Fundo, faculdade de educação física e fisioterapia, Passa Fundo, 2013.

CORREAA, B.; SCHOTTEN, L.; MACHADO, Marcela. Aromaterapia na saúde e na beleza: desenvolvimento de um manual prático. Trabalho de Conclusão do Curso Superior de Tecnologia em Cosmetologia e Estética, Universidade do Vale do Itajaí, Balneário Camboriú, 2010.

COSTA, Adilson; ALCHORNE, Maurício Motta de Avelar; GOLDSCHMIDT, Maria Cristina Bezzan. Fatores etiopatogênicos da acne vulgar. Anais brasileiros de dermatologia, v. 83, p. 451-459, 2008.

CRUZ, Thamires Silva; DA PAIXÃO, Juliana Azevedo. Aplicação do Óleo Essencial de Melaleucaalternifolia (TEA TREE) no tratamento da acne vulgar. Revista Artigos. Com, v. 29, p. e7657-e7657, 2021. 
DE ARAÚJO, Ana Paula Serra; DELGADO, Daniela Cardoso; MARÇAL, Regiane. Acne diferentes tipologias e formas de tratamento. 2011.

DE OLIVEIRA, Aline Zulte; TORQUETTI, Camila Barbosa; DO NASCIMENTO, Laís Paula Ricardo. O TRATAMENTO DA ACNE ASSOCIADO À LIMPEZA DE PELE. Revista Brasileira Interdisciplinar de Saúde, 2020.

DIAS, Maria João Cunha. Impacto Psicossocial da ACNE: Influência do tratamento com isotretinoína. 2014.

Dini I, Laneri S. Nutricosmetics: A brief overview. Phytother Res. 2019 Dec;33(12):30543063. doi: 10.1002/ptr.6494. Epub 2019 Sep 3. PMID: 31478301.

ESTRELA, Jackelline Vieira et al. Efeito do led na flacidez tissular facial. CATUSSABAISSN 2237-3608, v. 3, n. 2, p. 29-36, 2014.

FERREIRA, Lilian Abreu; OLIVEIRA, Raiane Aparecida Gontijo. Os efeitos da camuflagem cosmética na qualidade de vida de pacientes com acne. Psicologia e Saúde em debate, v. 3, n. 2, p. 15-29, 2017.

Fried RG, Wechsler A. Psychologicalproblems in the acne patient. Dermatol Ther 19: 237240 (2006).

KALIL, Celia Luiza PetersenVitello; REINEHR, Clarissa Prieto Herman; DE MATTOS MILMA, Laura. Luz intensa pulsada: revisão das indicações clínicas. Surgical\&CosmeticDermatology, v. 9, n. 1, p. 9-16, 2017.

Kim RH, Armstrong, AW. Currentstateof acne treatment: highlighting lasers, photodynamictherapy, andchemicalpeels. Dermatol Online J. 2011;15(17):1-2.

Krejci-Manwaring J, Kerchner K, Feldman SR, Rapp DA, Rapp SR. Social sensitivityand acne: the role of personality in negative social consequencesandqualityof life. Int J Psychiatry Med. 2006;36(1):121-30. doi: 10.2190/FQXN-K610-BNY8-UQ0L. PMID: 16927583.

KURTALIĆ, Nermina et al. Qualidade de vida de adolescentes com acne vulgar. Acta medica Croatica: casopisHravatskeakademijemedicinskihznanosti , v. 64, n. 4, pág. 247$251,2010$.

LANGER, Laura Ingrid Volkweis; PRIM, Lucas Rodrigues. Investigação de depressão e qualidade de vida em pacientes em tratamento para acne. 2020. Trabalho de conclusão de curso, Faculdade Evangélica Mackenzie do Paraná, Curitiba, PR, 2020.

López-Estebaranz JL, Herranz-Pinto P, Dréno B; el grupo de dermatólogos expertos enacné. Consensus-Based Acne Classification System andTreatmentAlgorithm for Spain. ActasDermosifiliogr. 2017 Mar;108(2):120-131. English, Spanish.

MACHADO, Daiane Borges; SANTOS, Darci Neves dos. Suicídio no Brasil, de 2000 a 2012. Jornal Brasileiro de Psiquiatria, v. 64, p. 45-54, 2015. 
MONTAGNER, Suelen; COSTA, Adilson. Diretrizes modernas no tratamento da acne vulgar: da abordagem inicial à manutenção dos benefícios clínicos. Surgical\&CosmeticDermatology, v. 2, n. 3, p. 205-213, 2010.

NEVES, Catarina Rebelo. Acne e saúde pública: um contributo. 2016. Tese (Doutorado em ciências da vida na especialidade de saúde público)-Faculdade de Ciências Médicas da Universidade Nova de Lisboa, Lisboa, 2016.

NEVES, Catarina Rebelo et al. A vivência da Acne e as suas consequências psicológicas. Brazilian Journal of Health Review, v. 4, n. 1, p. 1266-1294, 2021.

ÖZTÜRK, Perihan et al. Assessment oftemperamentandcharacter profile withanxietyanddepression in patientswith acne. Balkan medical journal, v. 30, n. 2, p. 161, 2013.

OGE', L. K.; BROUSSARD, A.; MARSHALL, M. D. Acne Vulgaris: DiagnosisandTreatment. American familyphysician, v. 100, n. 8, p. 475-484, 2019.

PEREIRA, Jéssica Gomide; COSTA, Kleber França; DA ROCHA SOBRINHO, Hermínio Maurício. Acne vulgar: associações terapêuticas estéticas e farmacológicas. Revista Brasileira Militar de Ciências, v. 5, n. 13, 2019.

Peric S, Bubanj M, Bubanj S, Jancic S. Sideeffects assessment in glicolycacid peelings in patientswith acne type I. Bosn J Basic Med Sci. 2011;11(1):52-7.

PICARDI, Angelo; LEGA, Ilaria; TAROLLA, Emanuele. Suicide risk in skindisorders. Clinics in dermatology, v. 31, n. 1, p. 47-56, 2013.

PICARDI, Angelo; MAZZOTTI, Eva; PASQUINI, Paolo. Prevalenceand correlates ofsuicidalideationamongpatientswithskindisease. Journal of the American Academy of Dermatology, v. 54, n. 3, p. 420-426, 2006.

RAMOS, Renato Teodoro; FURTADO, Yvone Alves de Lima. Transtornos de ansiedade. Revista Brasileira de Medicina, v. 66, n. 11, p. 365-374, 2009.

Rapp DA, Brenes GA, Feldman SR, et al. Angerand acne: Implications for qualityoflife, patientsatisfactionandclinicalcare. Br J Dermatol 151 (1): 183-9 (2004).

RIBAS, Jonas; OLIVEIRA, Cláudia Marina PB. Acne vulgar e bem-estar em acadêmicos de medicina. Anais Brasileiros de Dermatologia, v. 83, p. 520-525, 2008.

SARAIVA, Karoline Michaely Nóbrega; DO EGYPTO, Lívio Vasconcelos. Impacto na saúde mental do paciente com acne grave/moderada em uso da isotretinoína oral: Estudo qualitativo. Research, Society and Development, v. 10, n. 9, p. e14710917770-e14710917770, 2021. 
SOUZA, Valéria Maria de. Ativos Dermatológicos; Guia de ativos dermatológicos utilizados na farmácia de manipulação para medicamentos farmacêuticos. São Paulo: PROMABOOKS, 2009.

Tan JK. Psychosocialimpactof acne vulgaris: eva- luatingtheevidence. SkinTherapyLett. 9 (7): 1-3 (2004).

TASOULA, Eleni et al. O impacto da acne vulgar na qualidade de vida e saúde psíquica em jovens adolescentes na Grécia: resultados de uma pesquisa populacional. Anais Brasileiros de Dermatologia, v. 87, n. 6, p. 862-869, 2012.

TASSINARY, João; SINIGAGLIA, Marialva; SINIGAGLIA, Giovanna. Raciocínio clinico aplicado à estética facial : com estudos de casos e material em realidade aumentada. Lajeado: Estética Experts, 2019.

TEIXEIRA, Márcia Almeida Galvão; FRANÇA, Emmanuel Rodrigues de. Mulheres adultas com acne: aspectos comportamentais, perfis hormonal e ultra-sonográfico ovariano. Revista Brasileira de Saúde Materno Infantil, v. 7, p. 39-44, 2007.

TEIXEIRA, Vera; VIEIRA, Ricardo; FIGUEIREDO, Américo. Impacto psicossocial da acne. Journal of the Portuguese Society ofDermatologyandVenereology, v. 70, n. 3, p. 291-291, 2012.

TONIOLLO, Marcelo Bighetti; TERADA, Andrea Sayuri Silveira Dias; JUNIOR, Enzo Melchior. Celulite facial em lábio inferior oriunda de acne inflamada: relato de caso. Revista da Faculdade de Odontologia-UPF, v. 21, n. 3, 2016.

Uhlenhake E, Yentzer BA, Feldman SR. Acne vulgarisanddepression: a retrospectiveexamination. J Cosmetic Dermatol 9: 59-63 (2010).

Vilar GN, Santos LA, Sobral Filho JF. Qualityoflife, self-esteemandpsychosocialfactors in adolescentswith acne vulgaris. AnBras Dermatol. 2015 Sep-Oct;90(5):622-9. doi: 10.1590/abd1806-4841.201533726. PMID: 26560206; PMCID: PMC4631226.

Como citar este artigo (Formato ABNT):

RESENDE, Luísna Gabriela Aguiar Lobo de; SILVA, Gabriel Cardoso Oliveira da; CALDAS, Érica Carvalho. O Impacto Psicossocial da Acne Vulgar. Id on Line Rev. Psic., Dezembro/2021, vol.15, n.58, p. 351-367, ISSN: 1981-1179.

Recebido: 08/12/2021;

Aceito 21/12/2021;

Publicado em: 30/12/2021. 